\title{
A chemical conjugate between HER2-targeting antibody fragment and Pseudomonas exotoxin A fragment demonstrates cytotoxic effects on HER2-expressing breast cancer cells
}

\author{
Sunju Lee ${ }^{1}$, Sangsu Park ${ }^{1}$, Minh Tan Nguyen ${ }^{1,2}$, Eunyoung Lee ${ }^{1}$, Julee Kim ${ }^{1}$, Sangki Baek ${ }^{1}$, Chong Jai Kim ${ }^{3}$, Yeon Jin Jang ${ }^{1}$ \\ E Han Choe , * $^{1,}$ \\ ${ }^{1}$ Department of Physiology, Bio-Medical Institute of Technology, University of Ulsan College of Medicine, Asan Medical Center, Seoul \\ 05505, Korea, ${ }^{2}$ Department of Biotechnology, NTT Hi-Tech Institute, Nguyen Tat Thanh University, Ho Chi Minh City 70000, Vietnam, \\ ${ }^{3}$ Department of Pathology, Asan-Minnesota Institute for Innovating Transplantation, University of Ulsan College of Medicine, Asan \\ Medical Center, Seoul 05505, Korea
}

Conventionally, immunotoxins have been produced as a single polypeptide from fused genes of an antibody fragment and a toxin. In this study, we adopted a unique approach of chemical conjugation of a toxin protein and an antibody fragment. The two genes were separately expressed in Escherichia coli and purified to high levels of purity. The two purified proteins were conjugated using a chemical linker. The advantage of this approach is its ability to overcome the problem of low recombinant immunotoxin production observed in some immunotoxins. Another advantage is that various combinations of immunotoxins can be prepared with fewer efforts, because the chemical conjugation of components is relatively simpler than the processes involved in cloning, expression, and purification of multiple immunotoxins. As a proof of concept, the scFv of trastuzumab and the PE24 fragment of Pseudomonas exotoxin A were separately produced using $E$. coli and then chemically crosslinked. The new immunotoxin was tested on four breast cancer cell lines variably expressing HER2. The chemically crosslinked immunotoxin exhibited cytotoxicity in proportion to the expression level of HER2. In conclusion, the present study revealed an alternative method of generating an immunotoxin that could effectively reduce the viability of HER2-expressing breast cancer cells. These results suggest the effectiveness of this method of immunotoxin crosslinking as a suitable alternative for producing immunotoxins. [BMB Reports 2019; 52(8): 496-501]

*Corresponding author. Tel: +82-2-3010-4292; Fax: +82-2-30108148; E-mail: hchoe@ulsan.ac.kr

https://doi.org/10.5483/BMBRep.2019.52.8.250

Received 5 November 2018, Revised 11 November 2018, Accepted 28 November 2018

Keywords: Breast cancer, Exotoxin, HER2, Pseudomonas, scFv

\section{INTRODUCTION}

Most anticancer monoclonal antibodies exhibit weak antibodydependent cytotoxic activity. A recombinant immunotoxin is a genetically engineered antibody fragment conjoined to a protein toxin that reduces the tumor tissues (1). The antibody region of these molecules specifically targets tumor cell surface receptors and then internalizes toward the endocytic compartment. Toxin molecules delivered to the cytosol of the target tumor cells destroy the target cells effectively $(2,3)$.

Human breast cancers are classified into subtypes depending on their gene expression patterns (4). The overexpression of human epidermal growth factor receptor 2 (HER2), also called HER2/neu, ERBB2, or CD340, has been observed in 20-30\% of all breast tumors (5). HER2, a 185-kDa transmembrane tyrosine kinase receptor, belongs to the epidermal growth receptor (EGFR) family 2. The phosphorylation of HER2 dimers results in the activation of various downstream processes, such as cell proliferation, survival, differentiation, angiogenesis, invasion, and metastasis (6). Patients with HER2 overexpression show a significantly poor prognosis and overexpression of HER2 in breast tissues stimulates malignant phenotypic transformation. In addition, HER2-overexpressing tumors are more resistant to general chemotherapy treatment (7).

Trastuzumab, an anti-HER2 antibody, has been approved by the FDA for the treatment of HER2-positive early-stage breast cancer and metastatic breast cancer (8). Trastuzumab blocks HER2 signaling by binding to its extracellular domain and attracts immune cells to tumor sites, resulting in the inhibition of tumor growth (9). Because trastuzumab itself showed weak antibody-dependent cell cytotoxicity, it has been conjugated with various chemical drugs to enhance cytotoxicity for active targeting against HER2-positive breast cancer cells (10).

Pseudomonas exotoxin A (PE) is a bacterial exotoxin from Pseudomonas aeruginosa that is expressed as a protein with 613 amino acids (a.a.), and comprises three functional domains (11). The receptor-binding domain la (1-252 a.a.) is

ISSN: 1976-670X (electronic edition)

Copyright (c) 2019 by the The Korean Society for Biochemistry and Molecular Biology

(c) This is an open-access article distributed under the terms of the Creative Commons Attribution Non-Commercial License (http://creativecommons.org/licenses/by-nc/4.0) which permits unrestricted non-commercial use, distribution, and reproduction in any medium, provided the original work is properly cited. 
followed by the translocation domain II (253-364 a.a.). The last four residues (400-404 a.a.) of domain Ib (365-404 a.a.) with domain III (405-613 a.a) is a catalytic subunit of the toxin (12). The catalytic enzyme activity of domain Ib and domain III ADP-ribosylates the elongation factor of the host ribosome, causing apoptotic cell death (13). The 40-, 38-, or $24-\mathrm{kDa}$ portions of the PE without the cell binding domain, designated as PE40, PE38, and PE24, respectively, was fused to the antibody fragment that targets the cancer cell (14).

In this study, we adopted a unique approach of chemical conjugation between an antibody fragment and a toxin instead of the traditional immunotoxins that are recombinant fusion proteins of the two proteins. An advantage of this approach is that it can overcome the problem of low recombinant immunotoxin production that is observed in some immunotoxins. As a proof of concept, the scFv of trastuzumab and the PE24 protein were produced separately using $E$. coli and then chemically crosslinked. The new immunotoxin was tested on the breast cancer cell lines that express HER2.

\section{RESULTS}

\section{Cloning the constructs}

To fuse three PCR products (i.e., $V_{H}, V_{L}$, and donor vector [pDONR207]) and create pENTR-HER2(scFv), an overlap cloning method was used. The primers were designed for PCR products to have homologous sequences at both the ends. After overlap cloning, the TEV cleavage site was added at the $\mathrm{N}$-terminal of HER2(scFv), and cysteine residue was added at the C-terminal for crosslinking reaction. A linker was inserted between $\mathrm{V}_{\mathrm{H}}$ and $\mathrm{V}_{\mathrm{L}}$. The attL1 or attL2 site was added at each terminal for the next cloning step, and the expression vector for MBP-HER2(scFv) was obtained using the LR reaction of the gateway cloning method with pENTR-HER2(scFv) and pDEST-HMGWA containing MBP tag (Fig. 1A, C). For making the PE24 expression vector, a multisite gateway cloning method was used. PE24-encoding gene was amplified by PCR. The attB1 and TEVrs sequence at the N-terminal and attB5 at the C-terminal of PE24 were added. attB site-flanked PE24 was inserted to the donor vector (pDONR221) by BP reaction and pENTR-PE24 was formed. The expression vector for His8-PE24 was created by LR reaction with His8 tag containing pDEST-His8 and pENTR-PE24 (Fig. 1B, D).

\section{Expression and solubility analysis of HER2(scFv) and PE24}

The expression vector for MBP-HER2(scFv) or His8-PE24 was transformed to $E$. coli BL21. The protein expression and solubility level were determined at different induction temperatures of $37^{\circ} \mathrm{C}$ or $18^{\circ} \mathrm{C}$.

E. coli was grown at $37^{\circ} \mathrm{C}$ until O. $D_{600}=0.6-0.7$. When the O.D value reached the optical value, $0.5 \mathrm{mM}$ IPTG was added and the protein expression was induced at $37^{\circ} \mathrm{C}$ for $3 \mathrm{~h}$ or $18^{\circ} \mathrm{C}$ for overnight. Then, the cells were sonicated. The total cell fraction, pellet, and soluble fraction were analyzed using

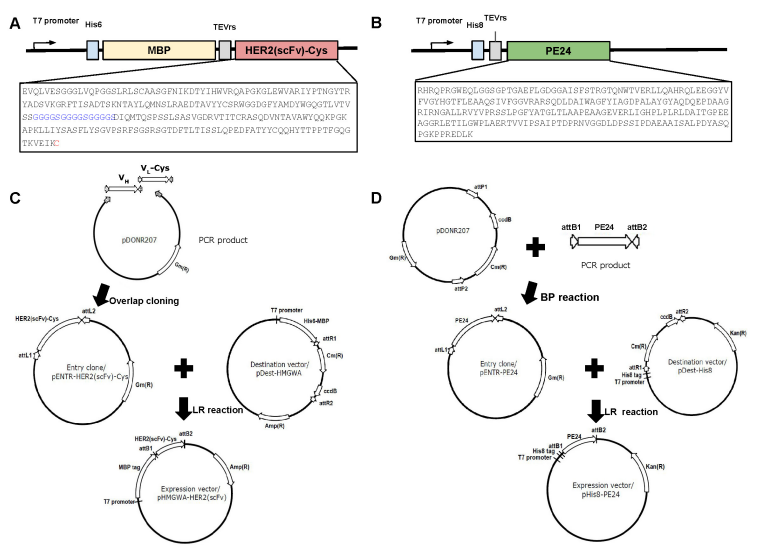

Fig. 1. Construct design and gateway cloning strategy of the expression vector. Designed constructs of (A) MBP-anti-HER2(scFv) and (B) His8-PE24. Cysteine residue was added at the C-terminal of anti-HER2(scFv) for crosslinking reaction. The TEV protease cleavage site was included at the N-terminal of both fusion proteins for tag removal. (C) MBP-HER2(scFv) expression vector was created by overlap cloning and gateway cloning methods. (D) The His8-PE24 expression vector was created by the gateway cloning method.

SDS-PAGE (Supplementary Fig. 1). MBP-HER2(scFv) and His8-PE24 fusion proteins were expressed at both the temperatures. However, when the proteins were induced at $18^{\circ} \mathrm{C}$, protein solubility was increased as compared with that at $37^{\circ} \mathrm{C}$ (Supplementary Table 1).

\section{Purification of HER2(scFv) and PE24}

The $E$. coli cells expressing MBP-HER2(scFv) were sonicated, and the soluble fraction of the cell lysate was applied to the HiTrap FF immobilized metal affinity chromatography (IMAC) column. The MBP-HER2(scFv) fusion protein was eluted at $100 \mathrm{mM}$ imidazole, and TEV protease was added to the elution containing MBP-HER2(scFv) at a ratio of 5:1 (fusion:TEV). After the MBP tag cleavage, HER2(scFv) was purified by the 2nd HiTrap FF IMAC column. The tag-free HER2(scFv) was collected from the flow through (FT) fraction, and the purified HER2(scFv) was dialyzed against phosphatebuffered saline (PBS) (Supplementary Fig. 2B). The yield of the final product was 31\% (Supplementary Table 2).

PE24 also was purified by IMAC chromatography, however, the His8-PE24 fusion protein was eluted at $500 \mathrm{mM}$ imidazole. TEV protease was treated to the eluted His8-PE24 at a ratio of 20:1 with $1 \mathrm{mM}$ DTT addition. After the TEV protease digestion, PE24 was purified from the FT fraction of the 2nd IMAC column in the same manner as HER2(scFv) (Supplementary Fig. 2C). After dialysis against PBS, the final yield was 39\% (Supplementary Table 3).

\section{Chemical conjugation of HER2(scFv) and PE24}

To generate an anti-HER2 immunoconjugate, anti-HER2(scFv) 
and PE24 were chemically conjugated via N-succinimidyl-3(2-pyridyldithio)propionate (SPDP), a disulfide bond-containing linker. The amino groups of PE24 were modified with SPDP (Fig. 2B, lane 1), and HER2(scFv)-Cys was reduced by TECP to make the sulfhydryl group available for conjugation (Fig. 2B, lane 2). After a reaction at a ratio of 5:1 (PE24:HER2(scFv)), the HER2(scFv)-PE24 conjugate was formed (Fig. 2B, lane 3). Then, this conjugate was purified by size exclusion chromatography using the Hiload 16/600 Superdex 75 pg (Fig. 2B, lane 4). The purity of the purified HER2(scFv)-PE24 conjugate was verified by SDS-PAGE under non-reducing condition $(10 \%$ Tricine gel). The highest yield and purity of the HER2(scFv)-PE24 conjugate was 58\% and 93\%, respectively (Supplementary Table 4).
A

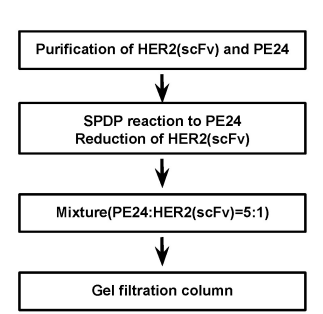

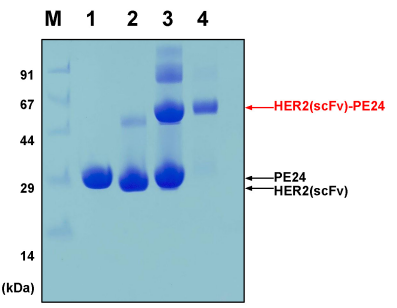

Fig. 2. Chemical conjugation with SPDP linker. (A) A schematic overview and (B) SDS-PAGE analysis of the crosslinking process and purification of the conjugated HER2(scFv)-PE24. Lane 1, SPDP-modified PE24; Lane 2, TECP-treated HER2(scFv); Lane 3, reaction mixture after incubation at $4^{\circ} \mathrm{C}$ for overnight; Lane 4, purified HER2(scFv)-PE24 conjugate after gel filtration column.
A

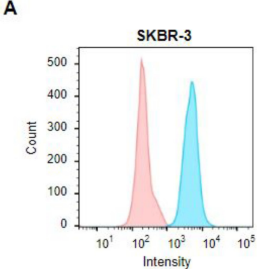

C

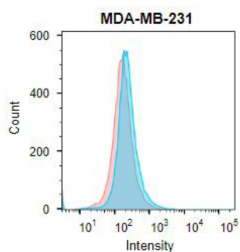

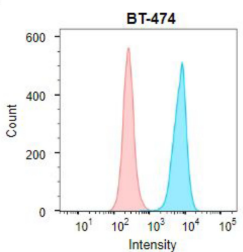

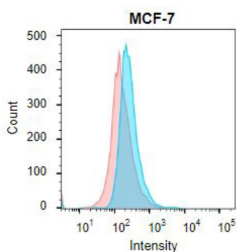

Fig. 3. Binding capacity of the HER2(scFv)-GFP conjugate. Flow cytometry analysis of HER2-overexpressing cell and HER2 low-expressing cell after incubation with DAPI (red) or DAPI and HER2(scFv)-GFP conjugate (blue). The fluorescence histograms indicate that HER2(scFv)-GFP strongly binds to HER2 receptors on (A) SKBR-3 and (B) BT-474 unlike HER2 low-expressing cell lines, (C) MDA-MB-231 and (D) MCF-7.

\section{HER2 expression of breast cancer cells}

To evaluate the binding capacity of the HER2(scFv)-PE24 conjugate, the HER2(scFv)-GFP conjugate was prepared and flow cytometry analysis was performed. After incubation with HER2(scFv)-GFP, a shift of the fluorescence histogram to the right was observed in the HER2-overexpressing cell lines, SKBR3 and BT-474 (Fig. 3A, B). On the contrary, the fluorescence histogram shifted slightly in HER2 low-expressing cells (MDA-MB-231, MCF-7) as compared with that in HER2-overexpressing cells (Fig. 3C, D). From the fluorescenceactivated cell sorting (FACS) analysis data, we determined that the HER2(scFv)-PE24 conjugate strongly binds to HER2expressing cells.

\section{Cytotoxicity of HER2(scFv)-PE24 conjugate in vitro}

To determine the cytotoxicity of the HER2(scFv)-PE24 conjugate, HER2-overexpressing and low-expressing cells were treated with HER2(scFv)-PE24, HER2(scFv), and PE24 at various concentrations. At $72 \mathrm{~h}$ of treatment, an MTT assay was performed to measure the cell viability. The HER2(scFv)PE24 conjugate revealed high toxicity in HER2-overexpressing cell lines (SKBR-3 and BT-474) at the picomolar level. The IC 50 value of SKBR-3 and BT-474 is $43 \mathrm{pM} \pm 8(\mathrm{n}=9)$ and $6.7 \mathrm{pM}$ $\pm 3 \mathrm{pM}(\mathrm{n}=9)$, respectively (Fig. $4 \mathrm{~A}, \mathrm{~B})$. In contrast, the HER2 low-expressing cell lines (MDA-MB-231 and MCF-7) were 20 - to $1.4 \times 10^{3}$-fold less affected by the HER2(scFv)-PE24 conjugate. The obtained $\mathrm{IC}_{50}$ values of MDA-MB-231 and MCF-7 were $9.44 \mathrm{nM} \pm 3 \mathrm{nM}(\mathrm{n}=9)$ and $1.01 \mathrm{nM} \pm 0.38$ $\mathrm{nM}(\mathrm{n}=9)$, respectively (Fig. $4 C, D)$. These data indicate that the cytotoxicity in cells is correlated with the HER2 expression
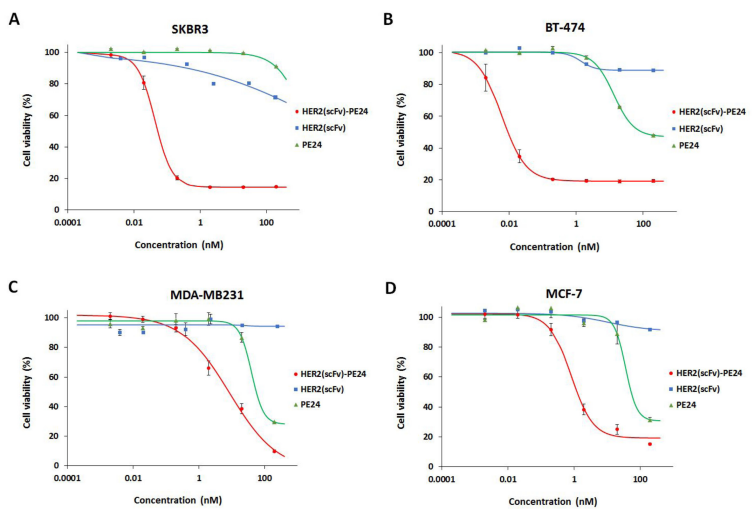

Fig. 4. Cell cytotoxicity of the HER2(scFv)-PE24 conjugate, HER2(scFv) and PE24. The cytotoxicity of the HER2(scFv)-PE24 conjugate was evaluated on HER2-overexpressing cell lines, SKBR-3 (A) and BT-474 (B), and HER2 low-expressing cell lines, MDA-MB-231 (C) and MCF-7 (D). The cell viability was measured by MTT assay, and the IC50 values of HER2(scFv)-PE24 conjugate were as follows: SKBR-3 (43 pM $\pm 8 \mathrm{pM}), \mathrm{BT}-474$ (6.7 $\mathrm{pM} \pm 3$ $\mathrm{pM})$, MDA-MB-231 (9.44 $\mathrm{nM} \pm 3 \mathrm{nM})$, and MCF-7 (1.01 $\mathrm{nM}^{ \pm} \pm$ $0.38 \mathrm{nM})$. 
on the cell surface. Treatment with HER2(scFv) alone did not inhibit the proliferation. Meanwhile, PE24 alone reduced cell viability at high concentration, except for the SKBR-3 cell line.

\section{DISCUSSION}

In this study, an antibody fragment and a toxin were produced separately from E. coli, and the two proteins were chemically conjugated using a chemical linker. An advantage of this approach is that it could overcome the low recombinant immunotoxin production problem observed in some immunotoxins. Another advantage is that various combinations of immunotoxins can be made with fewer efforts, because the chemical conjugation of the two components is simple. This was attempted previously for anti-CTLA-4 scFv and saporin (15), but has not been explored further.

Inside the cancer cell, the traditional recombinant immunotoxin is digested by the intracellular protease, furin, giving rise to dissociated antibody fragment and the toxin that interferes with intracellular function (16). The chemical crosslinker in our experiment creates a disulfide-containing linkage between the HER2(scFv) and PE24. Similar to furin cleavage site, the link would be cleaved inside the cell because of the reducing intracellular environment.

Four types of breast cancer cell lines were used to test the cytotoxicity of the chemically conjugated HER2(scFv)-PE24. SKBR-3 and BT-474 expressed HER2 higher than MDA-MB231 and MCF-7 did, as per the FACS analysis using HER2(scFv)GFP (Fig. 3). As expected, the cytotoxicity of the chemically conjugated immunotoxin was higher in SKBR-3 and BT-474 with $\mathrm{IC}_{50}$ of the picomolar range, whereas the other two cell lines showed $\mathrm{IC}_{50}$ of the nanomolar range (Fig. 4). This cytotoxicity of the chemically conjugated HER2(scFv)-PE24 on the four breast cancer cells was comparable to that of the conventional HER2(scFv)-PE24 (unpublished result), demonstrating the feasibility of the chemically conjugated immunotoxin. The efficacy of immunotoxins can be augmented by a hundred- or thousand-fold, but more than a million fold by endosomal escape enhancers in exceptional cases, such as lysosomotropic amines, carboxylic ionophores, calcium channel antagonists, various cell-penetrating peptides, other organic molecules, and light-induced techniques (17). Our immunotoxin already demonstrated $\mathrm{IC}_{50}$ of picomolar range, and it remains to be decided how much these endosomal escape enhancers could increase the efficacy.

In patients with solid tumors that have normal immune systems, $\mathrm{PE}$ is highly immunogenic because it is a bacterial protein. Anti-drug antibodies (ADA) were detected in all mesothelioma patients who were treated with recombinant immunotoxins (RIT) that contained PE (18). The ADA neutralized the RIT and prevented further treatment. Because immunocompromised hematological patients do not show a strong ADA response, a combination therapy of a RIT with an aggressive immunosuppression regimen was evaluated in patients with advanced chemo-resistant mesothelioma. ADA formation was delayed so that more cycles could be provided, and $40 \%$ of the patients showed dramatic tumor responses that substantially increased survival (19). This result demonstrated that RITs could induce major regressions in mesothelioma once immunogenicity was resolved and highlights the need to control immunogenicity to make therapy more effective.

PE38 was at the common fragment form of PE for recombinant immunotoxin (20). However, the nonspecific toxicity and strong immunogenicity of PE38 were the limitations of PE-based immunotoxins (21). For the prevention of immunogenicity, most of the domain II (253-364 a.a.) as B-cell and T-cell epitopes, except for the furin cleavage site, was removed. This fragment, PE24, significantly decreased immunogenicity and the nonspecific toxicity of PE (22). In addition, six amino acids of PE were identified as the B-cell epitope $(23,24)$. Six other amino acids of PE were mutated to decrease the immunogenicity of T-cell response $(22,25)$. Two amino acid positions were overlapped so that 10 amino acids were mutated for dual B- and T-cell de-immunization (26). Our PE24 was derived from 8 amino acid mutations to remove the B-cell epitope (27). Obviously, our PE toxin requires improvements in de-immunization.

Despite immunotoxins showing encouraging effects in clinical or preclinical animal trials, its short in vivo half-life hampers its therapeutic efficacy (28). There are several strategies to improve the pharmacokinetic properties of protein-based therapeutics, such as chemical modification with polyethylene glycol (PEGylation) or fatty acid, recombinant fusion with human serum albumin, albumin binding domain, or the Fc domain of the immunoglobulin (29). In a different approach for sustained release, protein or peptide drugs have been encaptured in the poly lactic-co-glycolic acid (PLGA) microsphere, phospholipid gel, or PEG gel (30). These methods may be applied to immunotoxins as well, in order to increase its in vivo half-life.

In conclusion, the present study represents another method of generating an immunotoxin. HER2(scFv) and PE24 were produced separately with high purities from $E$. coli. Then, the two proteins were chemically crosslinked. This immunotoxin effectively reduced the viability of HER2-expressing breast cancer cells. Our results suggest that this method of immunotoxin crosslinking is a good alternative to produce immunotoxins.

\section{MATERIALS AND METHODS}

\section{Construction of expression vector}

To create the expression vector for MBP-HER2(scFv), overlap cloning and multisite gateway cloning were performed. For the expression vector of His8-PE24, multisite gateway cloning method was used. More detail about these constructs is shown in the Supplementary Method. 


\section{Expression and solubility analysis of recombinant fusion protein in E. coli, BL21}

Expression plasmids were transformed into $E$. coli BL21 strain. E. coli was cultured and induced as mentioned in Supplementary Method 2. The fusion protein expression and solubility were analyzed with SDS-PAGE and assessed by an Image image analyzer (http://imagej.nih.gov.ij).

\section{Purification of HER2(scFv)-Cys and PE24}

HER2(scFv) and PE24 proteins were expressed in E. coli BL21 and purified by IMAC chromatography, as described in the Supplementary Method section.

Crosslinking and purification of HER2(scFv)-PE24 conjugate Crosslinking between HER2(scFv) and PE24 was performed using sulfo-LC-SPDP, as described in the Supplementary Method. The HER2(scFv)-PE24 conjugate was purified by gel filtration chromatography using the Hiload 16/600 Superdex 75 in PBS at pH 7.4 with 5 mM EDTA.

\section{Purification of GFP and HER2(scFv)-GFP conjugate}

HER2(scFv)-GFP was produced, as described in the Supplementary Method section.

\section{Flow Cytometric Analysis}

The cells were trypsinized and $2 \times 10^{6}$ cells were centrifuged and resuspended in $1 \mathrm{~mL}$ PBS. Then, $5 \mu \mathrm{g}$ of HER2(scFv)-GFP conjugate was added to each tube and incubated at $4{ }^{\circ} \mathrm{C}$ for 25 min. The cells were washed with PBS three times, and $1 \mu \mathrm{g}$ DAPI was added to each tube and incubated at $4^{\circ} \mathrm{C}$ for 10 min. After washing three times, the cells were analyzed by FACS Canto II flow cytometer (BD Biosciences, San Diego, CA). Flowjo V10 (Flowjo LLC, Ashland, OR) was used to analyze FACS data.

\section{In vitro cytotoxicity assay}

The SKBR-3, BT-474, MDA-MB231, and MCF-7 cells were grown in RPMI 1640 medium supplemented with $10 \%$ fetal bovine serum. The cells were seeded into 24-well plate at a density of $0.5-1 \times 10^{5}$ cells per well. After culturing for $24 \mathrm{~h}$, HER2(scFv)-PE24 conjugate was treated to seeded cell at different concentrations $(0.002,0.02,0.2,2,20$, and $200 \mathrm{nM})$. At $72 \mathrm{~h}$ of incubation with the conjugate, MTT assay was performed as described in the Supplementary Method.

\section{Statistics}

Data are presented as mean \pm standard error of $n \geq 3$ samples. The experiments were performed independently in triplicate.

\section{ACKNOWLEDGEMENTS}

This study was supported by grants from the National Research Foundation of Korea (NRF-2015K1A4A3046807 and 2017M
3A9F8031039) and a grant from the Asan Institute for Life Sciences, Seoul, Korea (2019-307).

\section{CONFLICTS OF INTEREST}

The authors have no conflicting interests.

\section{REFERENCES}

1. Mathew M and Verma RS (2009) Humanized immunotoxins: a new generation of immunotoxins for targeted cancer therapy. Cancer Sci 100, 1359-1365

2. Yamaizumi M, Mekada E, Uchida T and Okada Y (1978) One molecule of diphtheria toxin fragment $A$ introduced into a cell can kill the cell. Cell 15, 245-250

3. Antignani A and Fitzgerald D (2013) Immunotoxins: the role of the toxin. Toxins 5, 1486-1502

4. Goldhirsch A, Wood WC, Coates AS et al (2011) Strategies for subtypes--dealing with the diversity of breast cancer: highlights of the St. Gallen International Expert Consensus on the Primary Therapy of Early Breast Cancer 2011. Ann Oncol 22, 1736-1747

5. Perou CM, Sorlie T, Eisen MB et al (2000) Molecular portraits of human breast tumours. Nature 406, 747-752

6. Gutierrez C and Schiff R (2011) HER2: biology, detection, and clinical implications. Arch Pathol Lab Med 135, 5562

7. Carlomagno C, Perrone F, Gallo C et al (1996) c-erb B2 overexpression decreases the benefit of adjuvant tamoxifen in early-stage breast cancer without axillary lymph node metastases. J Clin Oncol 14, 2702-2708

8. Hudis CA (2007) Trastuzumab-mechanism of action and use in clinical practice. N Engl J Med 357, 39-51

9. Vu T and Claret FX (2012) Trastuzumab: updated mechanisms of action and resistance in breast cancer. Front Oncol 2, 62

10. Lambert JM and Berkenblit A (2018) Antibody-Drug Conjugates for Cancer Treatment. Ann Rev Med 69, 191-207

11. Wedekind JE, Trame CB, Dorywalska M et al (2001) Refined crystallographic structure of Pseudomonas aeruginosa exotoxin $A$ and its implications for the molecular mechanism of toxicity. J Mol Biol 314, 823-837

12. Siegall CB, Chaudhary VK, FitzGerald DJ and Pastan I (1989) Functional analysis of domains II, Ib, and III of Pseudomonas exotoxin. J Biol Chem 264, 14256-14261

13. Kreitman RJ, Arons E, Stetler-Stevenson M, Fitzgerald DJ, Wilson WH and Pastan I (2011) Recombinant immunotoxins and other therapies for relapsed/refractory hairy cell leukemia. Leuk Lymphoma 52 Suppl 2, 82-86

14. Weidle UH, Tiefenthaler G, Schiller C, Weiss EH, Georges G and Brinkmann U (2014) Prospects of bacterial and plant protein-based immunotoxins for treatment of cancer. Cancer Genomics Proteomics 11, 25-38

15. Tazzari PL, Polito L, Bolognesi A et al (2001) Immunotoxins containing recombinant anti-CTLA-4 single-chain fragment variable antibodies and saporin: in vitro results and in vivo effects in an acute rejection model. J Immunol 167, 4222-4229 
16. Weldon JE, Xiang L, Zhang J et al (2013) A recombinant immunotoxin against the tumor-associated antigen mesothelin reengineered for high activity, low off-target toxicity, and reduced antigenicity. Mol Cancer Ther 12, 48-57

17. Fuchs $H$, Weng $A$ and Gilabert-Oriol $R$ (2016) Augmenting the Efficacy of Immunotoxins and Other Targeted Protein Toxins by Endosomal Escape Enhancers. Toxins 8, 200

18. Hassan R, Bullock S, Premkumar A et al (2007) Phase I study of SS1P, a recombinant anti-mesothelin immunotoxin given as a bolus I.V. infusion to patients with mesothelin-expressing mesothelioma, ovarian, and pancreatic cancers. Clin Cancer Res 13, 5144-5149

19. Hassan R, Miller AC, Sharon E et al (2013) Major cancer regressions in mesothelioma after treatment with an anti-mesothelin immunotoxin and immune suppression. Sci Transl Med 5, 208ra147

20. Kreitman RJ, Siegall CB, Chaudhary VK, FitzGerald DJ and Pastan I (1992) Properties of chimeric toxins with two recognition domains: interleukin 6 and transforming growth factor alpha at different locations in Pseudomonas exotoxin. Bioconjug Chem 3, 63-68

21. Hassan R, Alewine C and Pastan I (2016) New Life for Immunotoxin Cancer Therapy. Clin Cancer Res 22, 1055-1058

22. Mazor R, Eberle JA, Hu X et al (2014) Recombinant immunotoxin for cancer treatment with low immunogenicity by identification and silencing of human T-cell epitopes. Proc Natl Acad Sci U S A 111, 8571-8576

23. Liu W, Onda M, Lee B et al (2012) Recombinant immunotoxin engineered for low immunogenicity and antigenicity by identifying and silencing human B-cell epitopes. Proc Natl Acad Sci U S A 109, 11782- 11787

24. Hu X, Zhang M, Zhang C et al (2016) Removal of B-cell epitopes for decreasing immunogenicity in recombinant immunotoxin against B-cell malignancies. J BUON 21, 1374-1378

25. Mazor R, Zhang J, Xiang L et al (2015) Recombinant Immunotoxin with T-cell Epitope Mutations That Greatly Reduce Immunogenicity for Treatment of MesothelinExpressing Tumors. Mol Cancer Ther 14, 2789-2796

26. Mazor R, Onda M, Park D et al (2016) Dual B- and T-cell de-immunization of recombinant immunotoxin targeting mesothelin with high cytotoxic activity. Oncotarget 7, 29916-29926

27. Onda M, Beers R, Xiang $L$ et al (2011) Recombinant immunotoxin against B-cell malignancies with no immunogenicity in mice by removal of B-cell epitopes. Proc Natl Acad Sci U S A 108, 5742-5747

28. Thurber GM, Schmidt MM and Wittrup KD (2008) Antibody tumor penetration: transport opposed by systemic and antigen-mediated clearance. Adv Drug Deliv Rev 60, 1421-1434

29. Wang $L$ and Ying $T$ (2016) New Directions for Half-Life Extension of Protein Therapeutics: The Rise of Antibody Fc Domains and Fragments. Curr Pharm Biotechnol 17, 1348-1352

30. Vaishya R, Khurana V, Patel S and Mitra AK (2015) Long-term delivery of protein therapeutics. Expert Opin Drug Deliv 12, 415-440 